\title{
Arbeitsbedingungen für Unterassistentinnen und -assistenten
}

Verband Schweizer Medizinstudierender

Die Gestaltung der Unterassistentenzeit ist in den einzelnen Spitälern der Schweiz sehr unterschiedlich. Der Verband Schweizer Medizinstudierender (VSM) möchte mit diesem Beitrag einige Punkte formulieren betreffend Arbeitsbedingungen der Unterassistentinnen und -assistenten und damit auch eine schweizweite Vereinheitlichung fördern und unterstützen. Dabei liegt das Schwergewicht auf folgenden Punkten, die für uns Studierende von besonderer Wertigkeit sind.

\section{Das Teaching}

Unterassistentinnen und -assistenten stehen kurz vor dem Abschluss ihres Studiums und werden danach als Assistenzärztinnen und -ärzte selbständig auf einer Abteilung in einem Spital arbeiten. Der VSM möchte darauf hinweisen, dass die Studierenden in ihrem vorletzten oder letzten klinischen Jahr als Lernende optimal gefördert werden sollten.

Das Teaching sollte im Mittelpunkt der Unterassistenzzeit stehen, d.h. qualitativ wie quantitativ so stattfinden, dass die zukünftigen Ärzte befähigt werden, ihren Beruf später entsprechend den an sie gestellten - zu recht sehr hohen - Anforderungen auszuüben.

Dies wird nicht zuletzt den ausbildenden Spitälern zugute kommen, an denen die Assistenzärztinnen und -ärzte später arbeiten werden.

\section{Die Entlöhnung}

Es ist nach Ansicht des VSM anzustreben, dass eine gesamtschweizerische Vereinheitlichung der Entschädigung/Entlöhnung für Unterassistentinnen und -assistenten erreicht wird.

Der VSM empfiehlt, dass die Entschädigung/Entlöhnung den Aufwand für Kost und Logis in keinem Fall unterschreitet (Basis: Zimmer im Personalhaus inkl. NK + Morgen-, Mittag- und Nachtessen im Personalrestaurant).

Wir halten dies für notwendig und auch gerechtfertigt, da die Unterassistentinnen und -assistenten für viele Spitäler nicht zu entbehrende Arbeitskräfte darstellen. Auf Anfrage des VSM gab das Staatssekretariat für Wirtschaft (seco) im Januar 2003 eine Stellungnahme zur Gültigkeit des Arbeitsgesetzes (ArG) für Unterassistentinnen und -assistenten ab. Hieraus werden einige Passagen zitiert:
«Unterassistenzärztinnen und -ärzte (cand. med. im Wahlstudienjahr) sind Arbeitnehmerinnen oder Arbeitnehmer, die im Rahmen ihrer Hochschulausbildung während einer bestimmten Zeit in einem Spital tätig sind. Diese Tätigkeit ist mit der Aufgabe eines Praktikanten oder einer Ausbildung im Betrieb gleichzustellen. Demzufolge fallen Unterassistenzärztinnen und -ärzte unter den persönlichen Geltungsbereich des Arbeitsgesetzes nach Art. 1 ArG (SR 822.11; ArG) und Art. 1 ArGV 1.»

\section{Zusammenfassung}

Der VSM möchte sich für einen guten klinischen Unterricht, wie er von vielen Spitälern jetzt schon geleistet wird, einsetzen. Der Zweck dieses Beitrages ist, dort eine Verbesserung der Lehre zu initiieren, wo diese noch nicht so optimal gestaltet ist. Wir möchten Sie hiermit bitten, sich für das Curriculum der Unterassistentinnen und -assistenten an Ihrem Spital nachhaltig einzusetzen.

Zudem ist es für den VSM anzustreben, dass die Unterassistentinnen und -assistenten für ihre Mitarbeit im Routinebetrieb und in den Nachtdiensten angemessen entschädigt werden.

Der VSM will schliesslich erreichen, dass das Arbeitsverhältnis zwischen Unterassistentinnen und -assistenten und den beschäftigenden Spitälern soweit wie möglich einheitlich geregelt wird und auf eindeutiger rechtlicher Basis beruht. Dabei darf nicht ausser acht gelassen werden, dass Unterassistentinnen und -assistenten dem ArG unterstellt sind. Entsprechend müssen die Bedingungen für ihre Arbeit ausgestaltet werden.

Wir hoffen, Ihnen mit unseren Ausführungen unser Anliegen ausreichend dargelegt zu haben: Wir brauchen gut ausgebildete Ärztinnen und Ärzte! Bitte helfen Sie mit, dieses Ziel zu erreichen, indem Sie an Ihrem Spital der Ausbildung der Unterassistentinnen und -assistenten einen besonders hohen Stellenwert einräumen und günstige Arbeitsbedingungen schaffen bzw. erhalten. 